\title{
Prevalence and outcomes of acute kidney injury in term neonates with perinatal asphyxia
}

\author{
Dan Alaro ${ }^{1}$, Admani Bashir ${ }^{1}$, Rachel Musoke ${ }^{1}$, Lucy Wanaiana ${ }^{1}$
}

1. Department of Paediatrics and Child Health, University of Nairobi.

2. Department of Paediatrics, The Aga Khan University, Nairobi.

\begin{abstract}
Background: The kidney is the most damaged organ in asphyxiated full-term infants. The severity of its damage is correlated with the severity of neurological damage. We determined the prevalence of perinatal asphyxia-associated acute kidney injury (AKI).

Methods: We conducted a prospective cohort study including 60 full-term neonates admitted at the Kenyatta National Hospital newborn unit (NBU) in Nairobi with hypoxic ischaemic encephalopathy (HIE) from June 2012 to November 2012. Renal function was assessed by measuring serum creatinine on day 3 of life. AKI was defined by a level of creatinine above $133 \mu \mathrm{mol} / \mathrm{l}$. The degree of neurological impairment was determined daily until patient discharge, death or day 7 of life.

Results: Of the 60 infants 36.6\% had HIE I, 51.6\% HIE II and 11.8\% HIE III. The prevalence of AKI was $11.7 \%$. There was a 15 fold increase risk of developing AKI in HIE III versus HIE I, $\mathrm{p}=0.034$. Mortality rate in perinatal asphyxia associated AKI was $71.4 \%$ with a 24 fold increase risk of death in neonates with AKI, $\mathrm{p}=0.001$.

Conclusions: AKI is common and associated with poorer outcomes in perinatal asphyxia. Larger studies need to be done to correlate maternal factors and perinatal asphyxia-associated AKI.

Key words: moderate perinatal asphyxia, severe perinatal asphyxia, hypoxic ischaemic encephalopathy, neonatal kidney dysfunction, neonatal neurological dysfunction.
\end{abstract}

DOI: http://dx.doi.org/10.4314/ahs.v14i3.26

\section{Introduction}

Perinatal asphyxia is defined by the World Health Organisation (WHO) as "Failure to initiate and sustain breathing at birth."(1) There is a high incidence of AKI among the asphyxiated term infants, $(50-72 \%)$. $(2,3)$. The presence of perinatal asphyxia and its severity appear to correlate with increasing incidence of AKI. $(2,4)$ Asphyxia is an important cause of AKI and transient kidney impairment with adverse effects, especially in the five days of birth. $(5,6)$ The kidney is the most damaged organ in asphyxiated full- term infants. (6) There lacks an internationally acceptable definition of AKI in neonates. Most of the previous investigators have defined AKI in neonates as serum creatinine above $133 \mu \mathrm{mol} /(1.7,8)$

\section{Corresponding author: \\ Dr. Dan Alaro \\ Department of Paediatrics and Child Health, University of Nairobi Email: danalaro@gmail.com}

The objective of this study was to determine the prevalence of acute kidney injury using serum creatinine above $133 \mu \mathrm{mol} / 1$ and short term outcomes of moderate to severe perinatal asphyxia-associated acute kidney injury.

\section{Methods}

This was a prospective cohort conducted at the $\mathrm{KNH}$ NBU over a 6 month period (June 2012 to November 2012). All term neonates with moderate to severe perinatal asphyxia admitted within $24 \mathrm{hrs}$ of delivery into the NBU. The 56 consecutive term newborns that satisfied the inclusion criteria were enrolled after written informed consent was obtained from either of the parents having given them clear explanation of the purpose of the study, expected benefits and potential harms. Ethical approval was obtained from the Ethical Review Committee, KNH. Newborns with malformation syndromes and those who died within 3 days of admission were excluded from the study.

Maternal and neonatal data was captured using pre-tested questionnaires and forms. Perinatal asphyxia was defined as failure to initiate and sustain breathing at birth (1), clinical evidence of HIE (9) and Apgar score less than 7 at 5 minutes. Gestational age was determined us- 
ing the Finnström score.(10)

Emergency care and resuscitation was a priority to any other procedures. Study details were given to the immediate caregivers. No beneficial treatment was withheld from the study subjects. All information about the patient was treated with the strictest confidence.

The neonate's length, body weight and head circumference were measured on admission. Neonatal body weight and the degree of neurological impairment were determined according to Sarnat classification daily until patient discharge, death or day

7 of life. Serum creatinine values were determined on day 2 and 3 of life by heel sampling of $0.5 \mathrm{ml}$ to $1 \mathrm{ml}$ into a microtainer. The sample was then centrifuged within 2 hours and analysed using Cobas Integra machine using the compensated Jaff $\square$ method.

\section{Statistical analysis}

Statistical analysis was conducted using statistical products and services solutions (SPSS) software version
17.0. Descriptive data were presented as numbers (percentages) or mean (standard deviation), as appropriate. Characteristics were compared using Fischer's exact test for dichotomous variables and Wilcoxon rank-sum test for continuous variables. Test of associations between the neonatal/ maternal factors and the study outcomes were performed using Chi-square test for categorical variables and comparisons of means and medians was done using student's test and Mann Whitney $\mathrm{U}$ test respectively. A p-value of 0.05 was considered statistically significant.

\section{Results}

Of the 968 newborns admitted into the KNH NBU during the study period, 60 infants met the inclusion criteria and were analysed as shown in figure 1.

The mean weight was $3373 \mathrm{~g}(\mathrm{SD}=427.3)$, length 52.3 $\mathrm{cm}(\mathrm{SD}=2.1)$, clinical gestation 39.4 weeks $(\mathrm{SD}=0.87)$ and head circumference $34.8 \mathrm{~cm}(\mathrm{SD}=1.6)$. The neonates weight ranged from 2620 to $4600 \mathrm{~g}$. Only $9 \%$ of the neonates admitted had a severe Apgar score (0-3), while $91 \%$ had a moderate Apgar score (4-6). Most of

\section{Figure 1: Flow chart showing the patient recruitment process}

Screened term neonates at NBU for perinatal asphyxia with subsequent HIE staging

Informed consent

Daily HIE Staging and serum creatinine on day 3 of life. AKI defined as serum creatinine level above $133 \mu \mathrm{mol} / 1$ at 72 hours of life.

24 Hourly followed up and clinically assessed for the first 7 days of life $(n=60)$

Data entered and analyzed for primary and secondary outcome $(n=60)$ 
the neonates were delivered in $\mathrm{KNH}(76 \%), 24 \%$ from other facility but none was delivered at home. Most of the neonates were delivered by the vaginal route $(63 \%)$, $33 \%$ via caesarian section and $4 \%$ via vacuum extraction. Only $40 \%$ of the neonates were resuscitated at birth and 18\% intubated and mechanically ventilated.

Majority of the mothers (86\%) were married and 53\% were primigravidae. Majority of the mothers were employed $(77 \%)$ versus $23 \%$ unemployed. Only $15 \%$ of the mothers had a level of education of primary school and below versus $75 \%$ who had attained secondary level of education and above. Almost all the mothers (90\%) reported having attended antenatal clinic (ANC) visit with $75 \%$ having attended ANC more than twice and $15 \%$ attending only once.
The degree of perinatal asphyxia was staged using Sarnat and Sarnat staging of Hypoxic Ischaemic Encephalopathy (HIE) on admission. Majority 31(52\%) had HIE II, $22(36 \%)$ HIE I and $7(12 \%)$ HIE III. Out of the neonates with AKI, $11.1 \%(4 / 36)$ of the males were affected versus $12.5 \%(3 / 24)$ of the females. 7 out of 60 neonates met criteria for AKI on day 3. Translates to a prevalence of $11.7 \%$

AKI was highest in the neonates with HIE 3 (42.9\%) on day 3 of life and lowest in the neonates with HIE 1 $(4.6 \%)$ as per figure 2 .

There was no statistically significant association between newborn, maternal characteristics and AKI (table 1 and 2).

Table 1: Tests of Association between Neonatal characteristics and AKI

Figure 2: Correlation between HIE and AKI

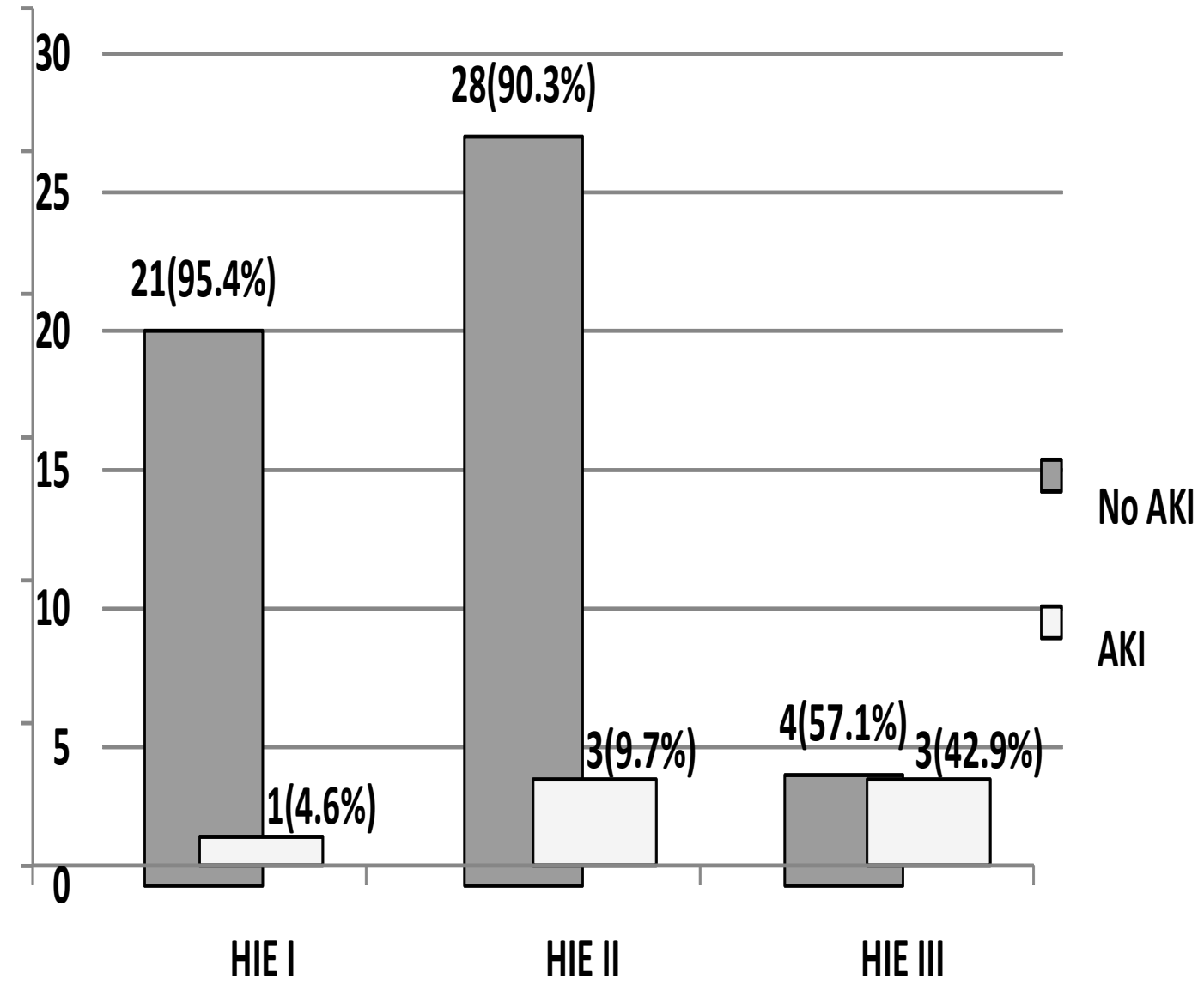


According to table 3 , the mortality rate in perinatal asphyxia associated AKI was $71.4 \%$. There was a 15 fold increase risk of developing AKI in HIE III compared to HIE I, $\mathrm{p}=0.034$ with $95 \% \mathrm{CI}(1.2-183.6)$. There was 24 fold increase risk of death in AKI, $\mathrm{p}=0.001$ with 95\% CI (3.7-157). Median day of death in neonates with AKI was 4.5 days
AKI $(n=7)$

Sex

Male

$4(57 \%)$

$3(43 \%)$

Moderate

$6(86 \%)$

$1(14 \%)$

Severe

$5(83 \%)$

$5(71 \%)$

No AKI $(n=53)$

$32(60 \%)$

1.000

Resuscitation

Place of delivery KNH

Other health facilities
$2(29 \%)$

$13(25 \%)$

Intubation $1(14 \%)$ $6(12 \%)$ 1.000

*Significant difference $(\mathrm{p}<0.05)$

The outcome of the neonates with AKI versus no AKI by day 7 of life was: discharged $14 \%$ versus $46 \%$, died $71 \%$ versus $9 \%$ and still admitted in NBU $14 \%$ ver- sus $30 \%$. The greater mortality rate $(71.4 \%)$ in the neonates with AKI may be high due to the small sample size number.

Discussion

Table 2: Tests of Ass ocia tion between Maternal charact eristics and AKI

\begin{tabular}{|c|c|c|c|c|}
\hline \multirow{2}{*}{\multicolumn{2}{|c|}{ Maternal Age $\{$ Median (IQR)\} }} & AKI $(n=7)$ & $\operatorname{NoAKI}(n=5)$ & Pvalue \\
\hline & & $29(19-33)$ & $27(24-29)$ & 0.630 \\
\hline \multicolumn{2}{|c|}{ Marital status Single } & $1(14 \%)$ & $7(13 \%)$ & 0.522 \\
\hline \multicolumn{2}{|c|}{ Married } & $6(86 \%)$ & $46(87 \%)$ & \\
\hline \multirow[t]{2}{*}{ Occupation } & bloyed & $4(57 \%)$ & $42(79 \%)$ & 0.075 \\
\hline & mployment & $3(43 \%)$ & $11(21 \%)$ & \\
\hline \multicolumn{2}{|l|}{ Maternal Fever } & $1(14 \%)$ & $3(6 \%)$ & 0.220 \\
\hline \multicolumn{2}{|l|}{$\mathrm{APH}$} & $1(14 \%)$ & $5(11 \%)$ & 1.000 \\
\hline \multirow[t]{2}{*}{ Level of education } & Primary and below & $2(29 \%)$ & $10(19 \%)$ & 0.330 \\
\hline & Secondary and above & $5(71 \%)$ & $43(81 \%)$ & \\
\hline \multirow[t]{4}{*}{ Mode of delivery } & Vertex Vaginal & $3(43 \%)$ & $34(64 \%)$ & 0.494 \\
\hline & Breech V aginal & $0(0 \%)$ & $1(2 \%)$ & \\
\hline & Caesarean & $4(57 \%)$ & $16(30 \%)$ & \\
\hline & Vacuum extraction & $0(0 \%)$ & $2(4 \%)$ & \\
\hline
\end{tabular}


According to the Acute Kidney Injury Network (AKIN), AKI is an absolute increase in serum creatinine of $\geq$ $26.4 \mu \mathrm{mol} / 1$ (or a percentage increase in serum creati- nine of at least $50 \%$ ) over two consecutive days. 7 Creatinine in the normal newborn at term by Schwartz is $79 \mu \mathrm{mol} / \mathrm{l}$ at day 1 and drops to $44 \mu \mathrm{mol} / \mathrm{l}$ ) at day

Table 3: Short-term outcomes of perinatal asphyxia associated AKI

\begin{tabular}{|c|c|c|c|c|}
\hline & $\operatorname{AKI}(\mathrm{N}=7)$ & No AKI $(n=53)$ & OD $(95 \% \mathrm{CI})$ & P Value \\
\hline HIE I & $1(14 \%)$ & $21(39 \%)$ & 1.00 & \\
\hline II & $3(43 \%)$ & $28(53 \%)$ & $2.22(0.2-23.0)$ & 0.5 \\
\hline III & $3(43 \%)$ & $4(8 \%)$ & $15(1.2-183.6)$ & $0.034 *$ \\
\hline Discharge in days $\{$ Median (IQR) $\}$ & $5(4-7)$ & $5(4-7)$ & & 0.56 \\
\hline Mortality & $5(71 \%)$ & $5(9 \%)$ & $24(3.7-157)$ & $0.001^{*}$ \\
\hline Time of death $\{$ Median (IQR)\} & $4.5(3.5-5)$ & $4(2-4)$ & & 0.45 \\
\hline
\end{tabular}

5.11 Before $48 \mathrm{~h}$ of life, the serum creatinine reflects that of the mother. 8

Studies by Jayshree 12, Nouri 14 and Gupta 2 chose the threshold of $90 \mu \mathrm{mol} / 1$ for serum creatinine at 48 hours of life. Studies by Karlowicz 3 Kaur 13 chose the serum creatinine threshold of $133 \mu \mathrm{mol} / 1$ at 48 hours to make a diagnosis of AKI. In our study, the threshold of $133 \mu \mathrm{mol} / 1$ for creatinine at 72 hours of life was chosen in order to increase our possibility of diagnosis as there would be a marked reduction in the maternal creatinine level by then.

There is a high incidence of AKI among the asphyxiated term infants $(7-72 \%)$. 2,3 AKI after perinatal asphyxia was noted in $42 \%$ of cases for Martin-Ancel 4 , $47 \%$ to Gupta 2, 68\% for Aggrawal $8,70 \%$ to Gluckman15, $17.2 \%$ Nouri14 and 33\% in our study. Our study noted $11.7 \%$ prevalence rate hence lying within the range of most of the studies done. The prevalence rate may have been much higher than the Tunisia study if serum creatinine was sampled at 48 hours and threshold of $90 \mu \mathrm{mol} / 1$ used. The available studies show that the prevalence rates were similar in both resource poor and resource rich areas proving that AKI in perinatal asphyxia is a global problem.

The presence of perinatal asphyxia and its severity appears to correlate with increasing incidence of AKI.2,4 Nouri14 who had two thirds of newborns with AKI had grade II and $1 / 3$ with AKI had grade III. However in his study no renal impairment was observed in newborns with grade I. The difference was not statistically significant $(p=0.13)$. Gupta 2 however showed that blood urea and serum creatinine were significantly higher in asphyxiated and HIE babies compared to the control group $(\mathrm{P}<0.001)$ and $(\mathrm{P}<0.05)$ respectively hence showing the correlation between AKI and HIE. Kaur13 showed that AKI developed in 1 out of 11 infants $(9.1 \%)$ with moderate asphyxia and in 12 of 25 $(56 \%)$ with severe asphyxia. Our study noted a 15 fold 
increase risk of developing AKI in HIE III compared to HIE I, $\mathrm{p}=0.034$ with $95 \%$ CI (1.2-183.6). However there was no correlation between HIE II and I $(p=0.50)$, and this could be explained by the small sample size. AKI was highest in the neonates with HIE 3 (42.9\%) on day 3 of life and lowest in the neonates with HIE 1 $(4.8 \%)$.

The small sample sizes in most studies have been a major hindrance in realizing associations between neonatal or maternal characteristics and AKI. Some authors have shown a significant association between low Apgar score at the 5th min and AKI, with a level of significance as low as $\mathrm{p}=0.0013$ by Nouri. 4,14 In our study $14 \%$ of the patients with severe Apgar score had AKI while majority $(86 \%)$ of the patients with moderate asphyxia had AKI. This could be explained by the fact that many of the patients who have severe asphyxia die before day 3 of life and hence are not included in the study. However, we found no significant correlation between Apgar and AKI ( $\mathrm{p}=0.473)$.

The mortality rates of perinatal asphyxia-associated AKI ranges between $2 \%$ and $20 \%(p=0.11) 2,14,13$ Our study revealed a $71.4 \%$ mortality rate $(p=0.001)$ and an average mortality at 4 days. There was 24 fold increase risk of death in AKI, $\mathrm{p}=0.001$ with $95 \%$ CI (3.7-157). The high mortality rate and wide confidence interval could have been contributed to by the small sample size. AKI is not normally a direct cause of death $^{16}$

The cause of death for patients diagnosed with AKI may not be the same as the cause of AKI. The mortality rate depends on other associated conditions, e.g. other organ failure, particularly cardiac failure, HIE and serious infection hence the difficulty in quoting the exact mortality rate secondary to AKI in our study and the previous ones.

1 out of every 8 neonates with moderate and severe perinatal asphyxia is likely to develop AKI with 5 out of 7 of these neonates likely to die by day 4 of life. AKI correlates with HIE, and the risk of developing AKI is higher with a more severe form of HIE. The neonates who have HIE III have a 15 times increased risk of developing AKI. The neonates who develop AKI have a 24 times increased risk of death. 1 out of 7 of the neonates who develop AKI will be discharged by day 7 of life. Clinicians should therefore endeavor to diagnose AKI and institute relevant measures from day 3 of life. Larger studies need to be done to correlate maternal factors and perinatal asphyxia- associated AKI.

\section{Acknowledgements}

University of Nairobi Department of Paediatrics Department of Research and Programs, Kenyatta National Hospital

\section{References}

1. World Health Organization. Basic Newborn resuscitation. A practical guide. World Health Organization; Geneva, 1997.

2. Gupta B, Pramod S, et al. The incidence of renal failure in asphyxiated neonates and to correlate severity and type of renal failure with Apgar score and hypoxic ischemic encephalopathy (HIE) grading of the neonates. Indian Pediatrics 2005; 42:928-934.

3. Karlowicz M, Adelman R. Nonoliguric and oliguric acute renal failure in asphyxiated term neonates. Pediatr Nephrol. 1995; 9:718-22.

4. Martin-Ancel A, Garcia-Alix A, Gaya F et al. Multiple organ involvement in perinatal asphyxia. J Pediatr 1995; 127: $786-793$.

5. Carter B, McNabb F. Prospective validation of a scoring system for predicting neonatal morbidity after acute perinatal asphyxia. J Pediatr.1998; 132: 619-23.

6. Adams-Chapman I, Stoll B. Nelson textbook of pediatrics. Philadelphia: WB Saunders; 2007. Nervous system disorders. p. 718.

7. Mehta R, Kellum J. Acute Kidney Injury Network (AKIN): report of an initiative to improve outcome in acute kidney injury. Crit Care 2007; 11:31-32.

8. Aggarwal A, Kumar P, Chowdhary G et al. Evaluation of renal functions in asphyxiated newborns. J Trop Pediatr 2005;51:295-9.

9. Aggarwal A, Kumar P, Chowdhary G, et al. Evaluation of renal functions in asphyxiated newborns. J Trop Pediatr 2005;51:295-9.

10. Finnström O. Studies on maturity in newborn infants. Acta Paediatr Scand 1977; 66:601-604.

11. Schwartz GJ, Feld LG, Langford DJ. A simple estimate of glomerular filtration rate in full-term infants falling on the first year of life. J Pediatr 1984; 104:84954.

12. Jayashree G, Dutta AK, Sarna MS, et al. Acute renal failure in asphyxiated newborns. Indian Pediatr 1991; 28:19-23.

13. Kaur S, Jain S. Evaluation of glomerular and tubular renal function in neonates with birth asphyxia. Ann Trop Paediatr. 2011; 31, 129-134.

14. Nouri S, Mahdhaoui N. Acute renal failure in full term neonates with perinatal asphyxia. Prospective study of 87 cases. Arch Pediatr. 2008; 15:229-235.

15. Gluckman P, Wyatt J, Azzopardi D, et al. Selective 
head cooling with mild systemic hypothermia Effective neonatal encephalopathy: multicentre randomized trial. Lancet 2005, 365: 663-670
16. Hoste EA, Kellum JA. Acute kidney injury: epidemiology and diagnostic criteria. Curr Opin Crit Care 2006; 12: 531-537. 